\title{
Data-Driven Manufacturing Simulation: Towards a CPS-Based Approach
}

\author{
Yongkuk JEONG ${ }^{\mathrm{a}, 1}$, Amita SINGH ${ }^{\mathrm{a}}$, Masoud ZAFARZADEH ${ }^{\mathrm{a}}$, \\ Magnus WIKTORSSON ${ }^{a}$ and Jannicke BAALSRUD HAUGE ${ }^{a}$ \\ ${ }^{a}$ KTH Royal Institute of Technology, Södertälje, Sweden
}

\begin{abstract}
Manufacturing simulation has been used as a decision support tool to solve various problems in production systems. However, with the advent of Industry 4.0 and CPS, manufacturing simulation becomes not only a tool for supporting decision-making but also essential for operation, monitoring, and forecasting the production system. In this paper, a traditional approach and a CPS-based approach in manufacturing simulation are compared. In the CPS-based approach, the key processes are divided into 1) data gathering, 2) modeling and simulation, and 3) simulation results analytics and feedback. In addition, a SWOT analysis is conducted to discuss the future application of the manufacturing simulation.
\end{abstract}

Keywords. Cyber-physical system, Manufacturing simuliation, SWOT analysis

\section{Introduction}

Manufacturing simulation refers to the computer-based modeling and analysis of real production systems. These are used to experimentally analyze, visualize and validate different possible setups for optimizing processes and supporting decision-making processes. With the fluctuating market demands and increasing product customization, it is imperative to have both reactive and proactive production systems. Hence, manufacturing simulation becomes an indispensable part of product development and industrialization in manufacturing by facilitating cost-effective solutions. In addition, with the advent of smart manufacturing and digital twin concepts, simulation is taking an important role in connecting the physical system and virtual system, supporting decision-making processes, predicting and controlling the production systems, and so on [1-3].

Traditional approaches in manufacturing simulation have the objective to support decision-making in strategic-planning, changes and investments of production systems. They are used for production planning, production line configuration, factory layout, and scenario comparison. In this approach, the simulation models are made for solving problems pertaining to a particular case resulting in single-use models. These models are typically based on the data from legacy systems, which are not completely synchronized leading to simulation models based on a set of assumptions [4]. Therefore, simulation

\footnotetext{
${ }^{1}$ Corresponding Author. yongkuk@kth.se
} 
models with the traditional approaches help in the developing or designing phase of the production system but are hardly used in the operational phase or all the life-cycle of the system.

The objective of this paper is to compare and discuss traditional approaches of the manufacturing simulation with the cyber-physical system-based (CPS-based) approach. This study is based on some previous literature presenting development trends and the future role of manufacturing simulation. Also, it discusses how the new technologies are changing the manufacturing simulation trend and makes a comparison study between traditional and CPS-based approach in manufacturing simulation.

\section{Method and approach}

The paradigm of manufacturing simulation was divided into four stages based on literature, and the main concept, modeling method, applications, and keywords of each stage were derived in this research. Also, smart manufacturing based on the digital twin and CPS has different characteristics from the traditional production system [4]. In this research, we compared the roles and features in terms of manufacturing simulation in traditional production systems and smart manufacturing. We divided the CPS-based approach into data gathering phase, modeling and simulation phase, simulation results analytics and feedback phase to analyze the characteristics based on the literature. Consequently, the basic process of data-driven manufacturing simulation is proposed and the strength, weakness, opportunity, and threats of the traditional and CPS-based approaches are analyzed through the SWOT analysis.

\section{Paradigm shift of the manufacturing simulation}

Simulation has been used in various fields such as product development, computational analysis, as well as manufacturing simulation of production systems. The role of simulation has been constantly changing with the paradigm shift. In the past, simulation was developed as a tool to help complex calculations. But with the digital twin and CPS paradigm, it has evolved into a decision support tool and system that covers the entire life-cycle and the system. This paradigm shift can be summarized with 1) individual application, 2) simulation tools, 3) simulation-based system design, and 4) digital twin concept as below in Figure 1.

The modeling paradigm of manufacturing simulation can be summarized with 1) computational simulation, 2) virtual factory, 3) digital factory, and 4) smart factory as shown in Table 1. Since 1960, simulation has been used for analyzing the results of different scenarios. The typical questions were often based on what-if analysis using scenario generation and changing variables. The data accuracy was often based on forecasts or experience and systematically used for planning purposes. By the mid-80s, simulations using three-dimensional visualization models appeared. It was used for collision detection simulation considering physical properties. Since 2000, the importance of information models has been emphasized, and many simulation systems have been developed to support decision-making processes. Also, simulation allows a 


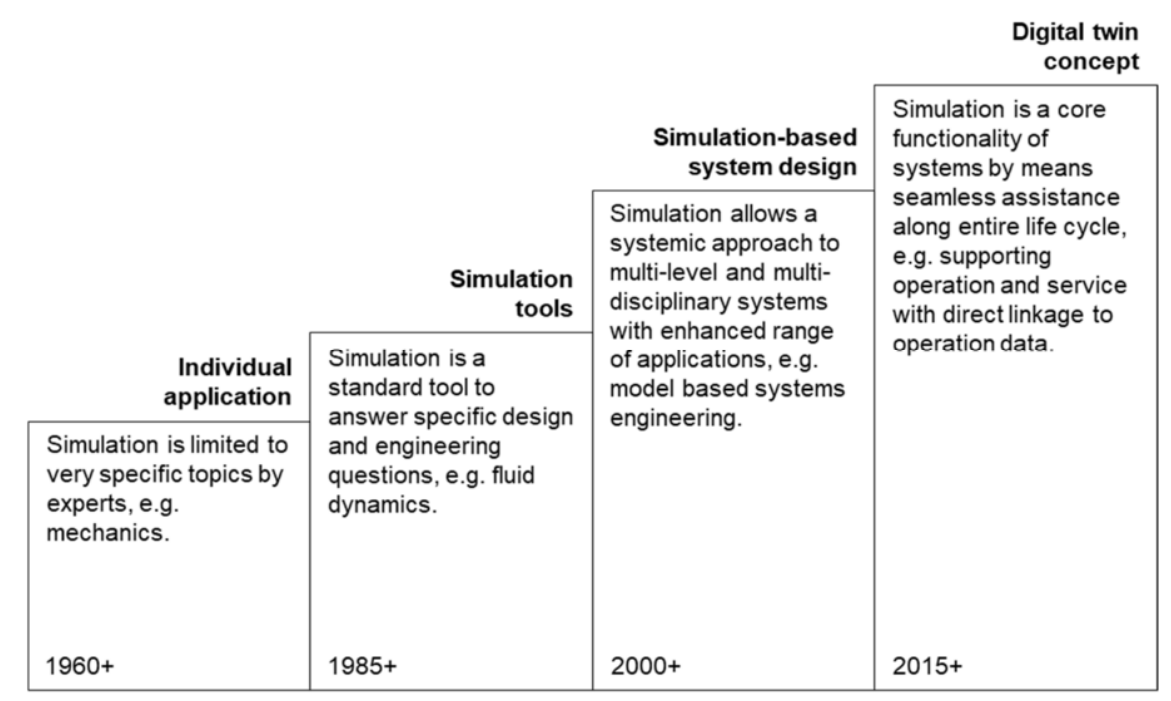

Figure 1 Paradigm shift of modeling and simulation (adapted from Rosen et al., 2015)

Table 1 Manufacturing simulation modeling paradigm

\begin{tabular}{|c|c|c|c|c|}
\hline Concept & $\begin{array}{c}\text { Computational } \\
\text { simulation }\end{array}$ & Virtual factory & Digital factory & Smart factory \\
\hline Period & $1960+$ & $1985+$ & $2000+$ & $2015+$ \\
\hline Model & $\begin{array}{c}\text { Computational } \\
\text { model for complex } \\
\text { calculation }\end{array}$ & $\begin{array}{c}\text { 3D virtualized } \\
\text { model }\end{array}$ & Information model & Feedback model \\
\hline Application & What-if simulation & Collision detection & $\begin{array}{c}\text { Decision support, } \\
\text { scheduling }\end{array}$ & CPS \\
\hline Keyword & What-if, scenario & 3D model & Digital, information & Smart, real-time \\
\hline
\end{tabular}

systematic approach to multi-level and multi-disciplinary systems with an enhanced range of applications. Around 2015, concepts such as Industry 4.0 and digital twin have emerged. Manufacturing simulation is used not only for operations but also for various fields such as control, monitoring, and forecasting using real-time data. Simulation is a core functionality of systems by means of seamless assistance along the entire life cycle.

In traditional production systems, it was difficult to collect data in real-time, and simulation models could not be constructed quickly. Therefore, simulation models are often used as a project to support decision-making in the long term. In this case, the historical data from the legacy system was used to collect the data, or the simulation engineer interviewed the operator or the site manager to define the input data. Consequently, manufacturing simulation models were developed for a single-use simulation project.

In contrast, in the CPS-based approach, a digital twin that simulates the physical world and utilizes synchronized data in real-time acts as a simulation model. Since the data is synchronized, real-time data can be used. Standard models and rapid simulation 
modeling techniques can also be used to support short-term decision-making as well as long-term perspectives of situations in the physical world. The changes of the role of manufacturing simulation can be described as Figure 2.

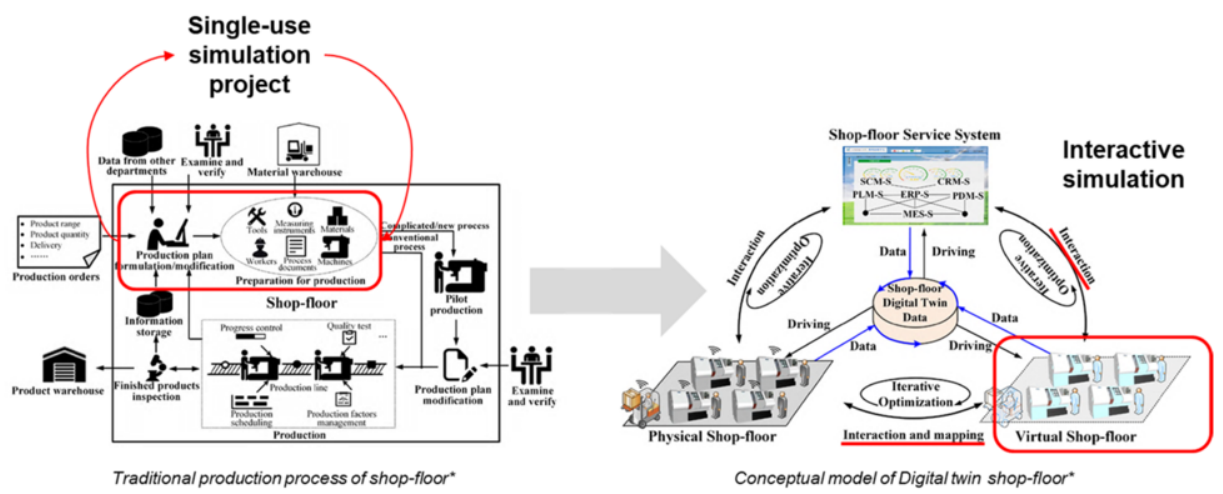

Figure 2 Changes in production systems and the role of manufacturing simulation (adapted from [4])

\section{Data-driven manufacturing simulation}

In this chapter, data-driven manufacturing simulation is divided into three phases and the characteristics of each phase are explained from the literature. The three phases are datagathering phase, modeling and simulation phase, simulation results analytics and feedback phase.

\subsection{Data-gathering}

Technologies for real-time data-gathering, as well as industrial Internet-of-Things (IoT) is rapidly expanding to be used within manufacturing, and enable seamless information flow as well as link information to the moving goods, manufacturing resources, and equipment. These technologies are not only a pre-requisite realizing concepts like Industry 4.0 and CPS [5], but also made it possible for the simulation to be more available as an operational tool to stakeholders.

Visibility is one of the main steps in the digitalization of the production field [6] and high visibility is a pre-requisite for fulfilling the Industry 4.0 vision, which foresees that sensors capture all data seamlessly and in real-time in each process, facilitating the interconnectivity of different sections and units [6]. Real-time data and improved visibility have revealed several challenges related to inefficient usage of resources, often caused by low transparency of movements and low degree of digitalization. 


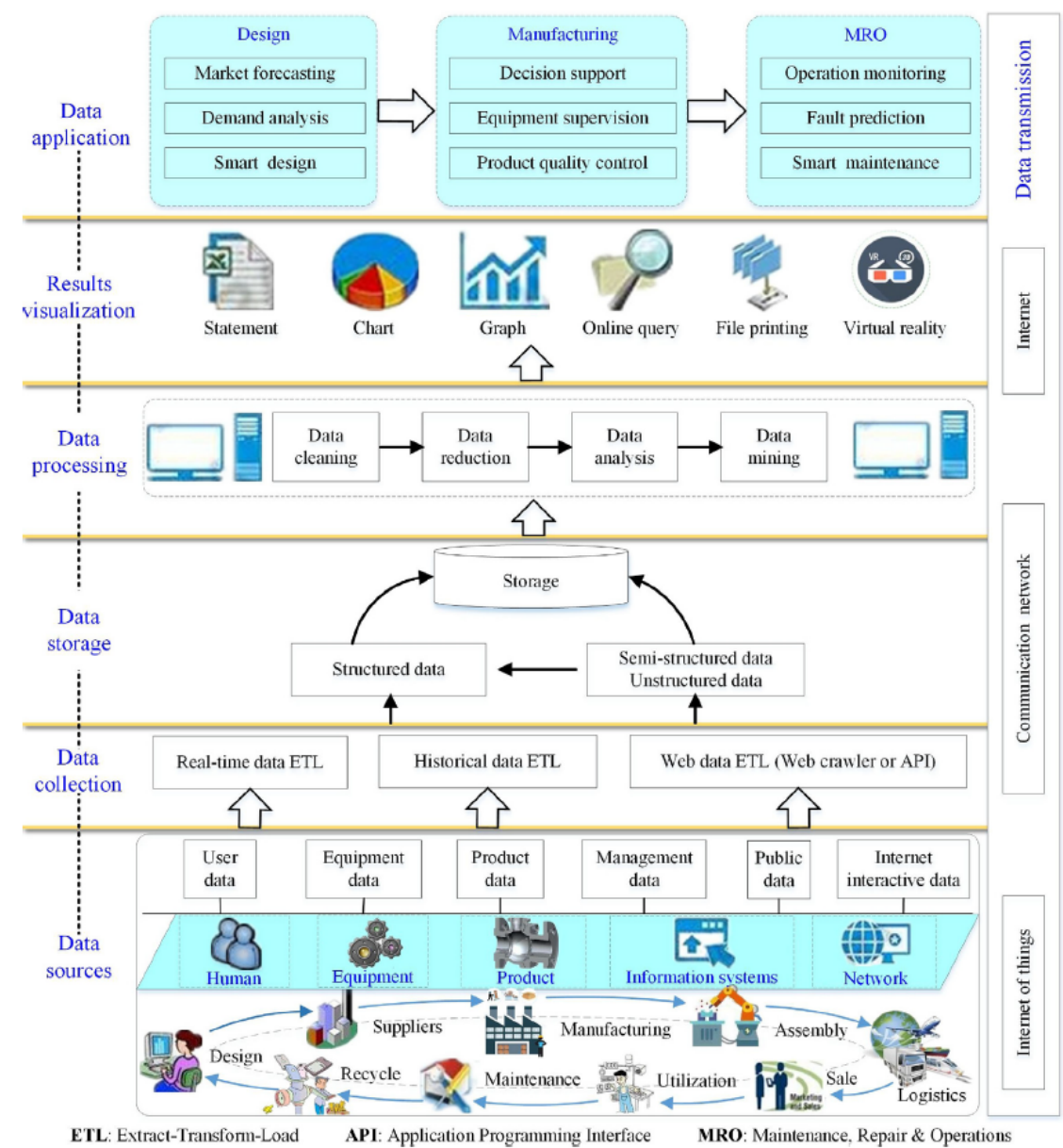

Figure 3 Manufacturing life cycle [7]

Figure 3 shows the life cycle of manufacturing data. Data can come from various types of sources like humans, equipment, product, information systems, and network. Means of data gathering can be IoT which collects data in real-time. Examples are Radio Frequency Identification (RFID), Real-time Locating System (RTLS), smart sensors and actuators. Sensors help to collect data regarding temperature, operational status, vibration, and pressure. RFID technology facilitates the automatic identification of parts. The location and position of items including human sources are possible through the implementation of RTLS. Developing different sort of Application Programming Interfaces (APIs) help to figure out the number and types of users, their location, etc. In case, data needs to be collected from public networks, web crawling can help by browsing public websites and collect the intended data [7]. 


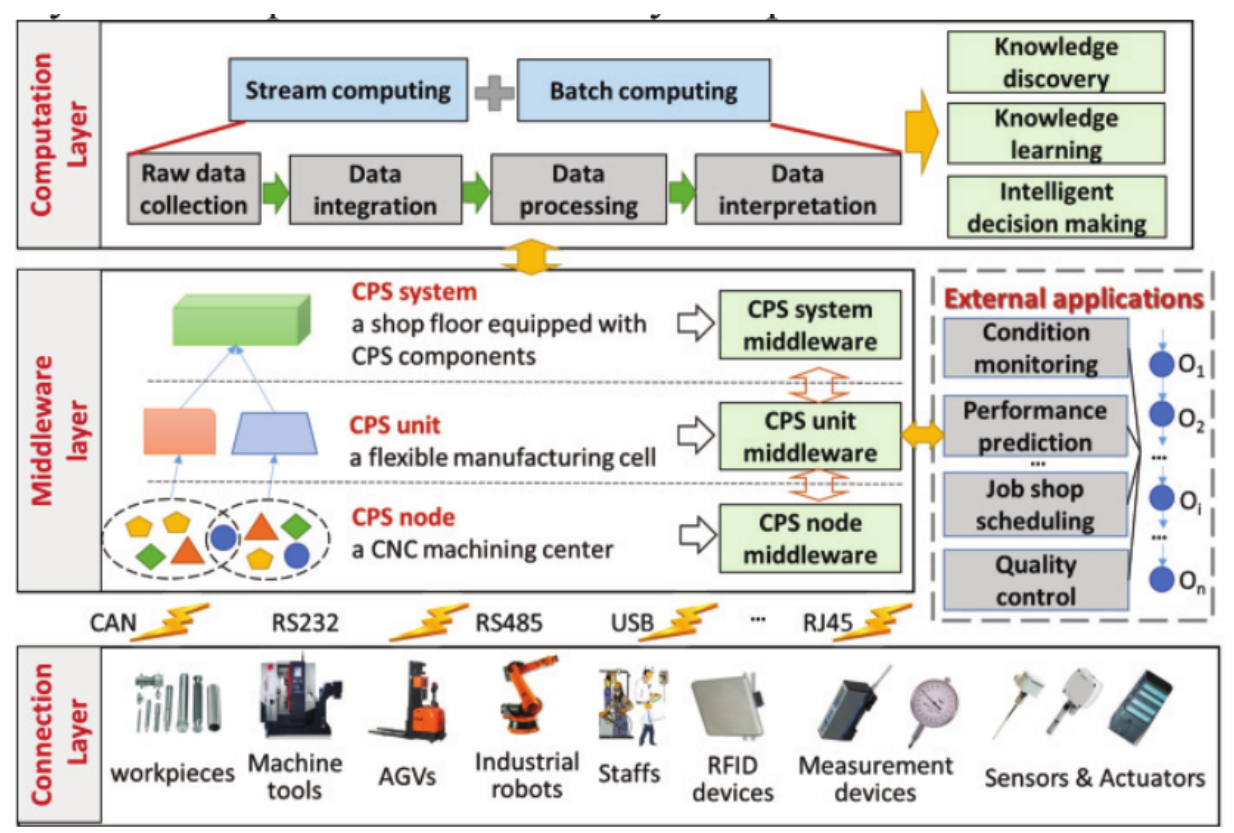

Figure 4 Data within CPS architecture for intelligent manufacturing [8]

In Figure 4, a three interconnected layers structure has presented where data are collected within a physical layer, go through a middle layer to transfer the data for further analysis and finally interpreted to create knowledge, support decision making, and knowledge learning [8]. Within the physical layer, after implementing technologies mentioned earlier, a group of machines is connected to each other through a filed bus or ethernet.

The middleware layer interconnects the connection layer, computational layer, and all the applications. As there might be different types of devices for data gathering which have their own protocol and standards, this layer supposed to facilitate smooth communication among all the devices. In addition, this layer should support interface definition by providing a channel for CPS node communication. On the other hand, a large amount of collected data needs to have a uniform format and unified data exchange standards.

The real-time data gathered from devices and historical data gathered from legacy systems such as Enterprise Resource Planning (ERP), Manufacturing Execution System (MES) provide input to the computational layer. Several algorithms, models and tools are required to find meaningful patterns and create knowledge and supporting information.

\subsection{Modeling and simulation}

In the CPS-based approach, the simulation model is no longer a static model. The simulation model is a dynamic model that is updated with real-time data. Models in the virtual world can be divided into physical models and manufacturing data collected from the physical world. An experimental digital twin is used as dynamic models through simulations using real-time data. It is described in Figure 5. 


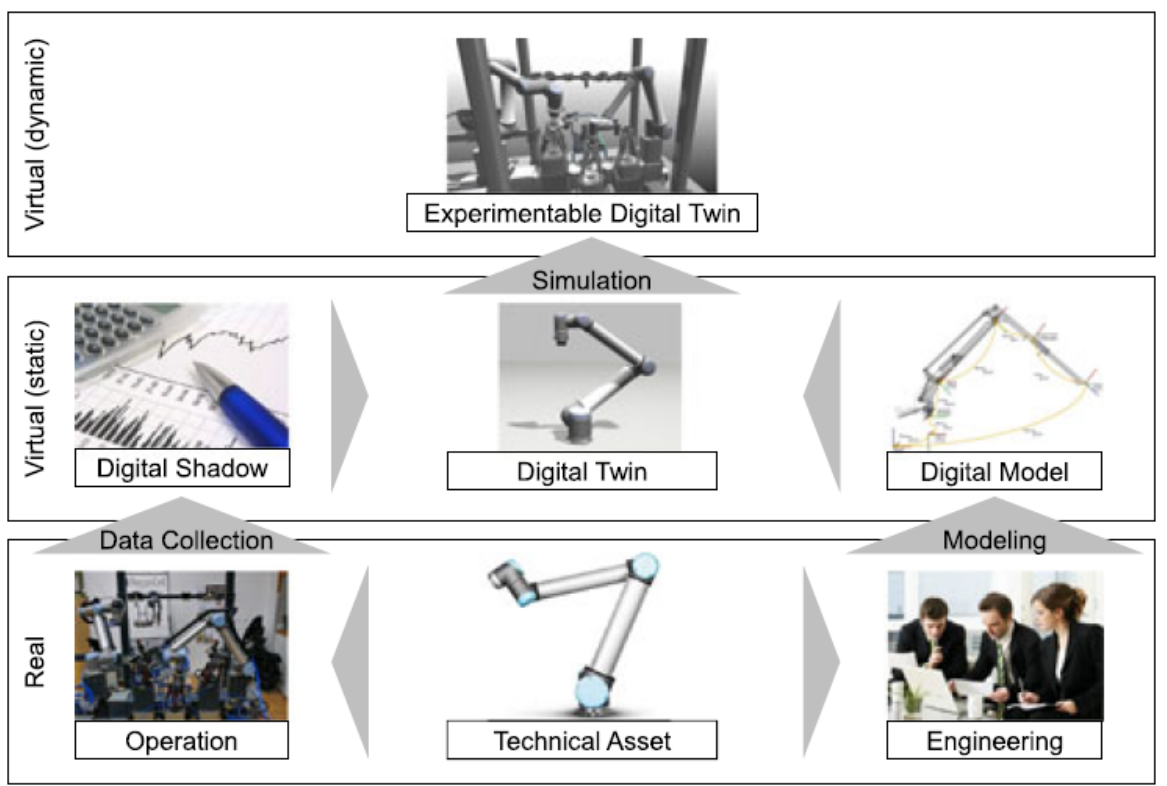

Figure 5 Dynamic digital twin model [9]

A standard model is required to develop a simulation model quickly and define a simulation model regardless of hardware or software. In order to solve this problem, Core Manufacturing Simulation Data (CMSD) was proposed [10, 11]. CMSD defines the features and relationships of core manufacturing entities. It helps to solve data interoperability issues in manufacturing simulations. CMSD uses UML and XML as modeling languages. It has a layout, part information, support, resource information, production operations, and production planning as its basic packages (Figure 6).

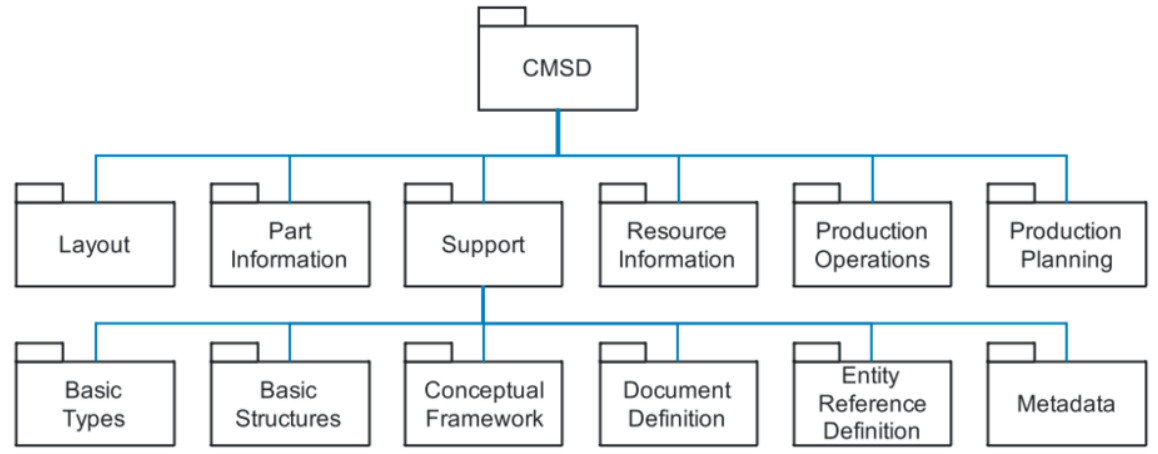

Figure 6 Basic packages of the CMSD model [11]

\subsection{Simulation results analytics and feedback}

With the help of insights data analytics can facilitate a better understanding of the production systems and their environments. There are advances being made in the field of sensor and industrial communication technologies used to collect data making it 
possible to accumulate big data from factories. Simultaneously, higher computational power is becoming available to process these data $[12,13]$. This has introduced some key differences in how analytics used to be done and how it is done now. In the remaining section, big data analytics and feedback to the real-world system in the context of production systems are discussed in detail.

Production systems are dynamic in nature with multiple criteria to be optimized in order to achieve an optimal solution. The data corresponding to a system is fed into a simulation model where analysis is done depending upon the criteria decided for a simulation model. As the number of variables to be optimized together with the data collected to go up in the model, the accuracy of the simulation results will be increased. Depending upon the complexity of a model in terms of how it maps to the real production system and the extent to which generalizations have been made, the accuracy of a model can be high or low. These approximations are used in the model while developing it and accordingly these systems are categorized in low and high fidelity models [14]. Low fidelity models use more approximations in terms of data used in a model and hence require less computational power. Thus, it can be seen as a tradeoff between computational resources required to build a model and accuracy of the model.

Results from data analytics are used twofold: 1) visualization of output data and 2) feedback to the physical real-world system.

Data visualization depends upon the requirement of a process at hand. The processed data can be visualized with the help of graphs, charts, dashboards, etc. and the data to be visualized can be customized to a particular need or purpose for engineers or shopfloor workers. Different devices like projectors, screens, tablets, smart glasses, etc. can be used to see the data. Smart factory-like setups uses such visualization techniques to help operators during their work [15].

In the second phase, results from data analysis are fed into physical systems in the real world. The results are used as input to machines and actuators in real-time resulting in a self-improvement process for the complete cyber-physical simulation model. Such a feedback model helps in short term prediction in dynamic environments leading to shorter decision-making times and also allowing reuse of simulation models as opposed to traditional ways of simulation where models were made with one objective and hence were single-use.

\section{Towards a CPS-based approach}

\subsection{CPS-based approach for manufacturing simluation}

CPS-based production system relies on data from sensors and embedded devices and based on the data provides feedback to the production systems. In this context, we have identified three major shifts concerning manufacturing simulation: 1) Real-time data gathering system, 2) big data availability and system interoperability enabling continuous input data availability and 3) advanced analytic application enabling system feedback and decentralized decision making.

Concerning the first shift, the sensors in the production system collect data resulting in data-driven simulation scenarios. The simulation model building is based on rapid building and parametric modeling. In contrast to the traditional approach of interviewing concerned people and reading documents, the CPS-based approach collects data and processes new simulation models. Regarding the second shift, the simulation models will 
increasingly be connected to other systems feeding real-time data and enabling iterations in a short period of time which makes it possible for the CPS-based simulation to be used also during the operational phase. For the last shift, the simulation model can provide feedback to the physical system and result in the self-improvement of production systems with better-fitted models. The production system autonomously adjusts itself with the changes in manufacturing units.

A basic process of the data-driven manufacturing simulation can be expressed as shown in Figure 7. First, manufacturing data is collected from various data sources in real-time and processed. Processed data can be used for monitoring the production system or visualizing to predict the state of the production system. In terms of simulation, models are developed using processed data. The simulation model can be divided into an information model, logic and algorithm, and object model. The model is defined using a standard data model. Simulation results are visualized through data analysis, and the simulation model can be reconfigured by itself. Simulation results can also be used to update the variables or properties of physical world components.

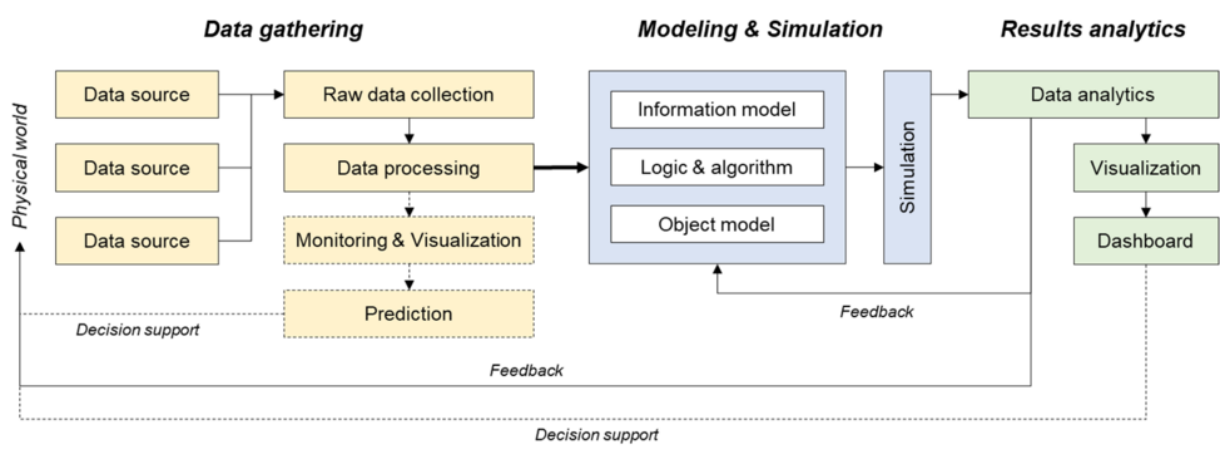

Figure 7 Basic process of data-driven manufacturing simulation

\subsection{SWOT anaylsis}

To compare the two different approaches discussed in this paper, a SWOT analysis was performed. First, the traditional approaches of the manufacturing simulation still have the advantage of high flexibility in a data collection process. It is one of the strengths that this approach can be used regardless of the level of automation of the target system or the infrastructure. However, as mentioned earlier, the weakness is that it is low in reusability and requires a lot of resources for the simulation project. Nevertheless, it can still be useful for SMEs or single-use projects with low levels of automation (Figure 8).

The strength of the CPS-based approach, which threatens traditional manufacturing simulations, can be seen as high reusability, predictive and self-configurable models. However, there is a weak point that this requires the construction of infrastructures such as a real-time data collection platform and data analysis platform. However, in the age of Industry 4.0, when many manufacturing systems have the infrastructure and automated systems it can be widely used in various fields. Cybersecurity, reliability of data and systems, and stability should be considered (Figure 9). 

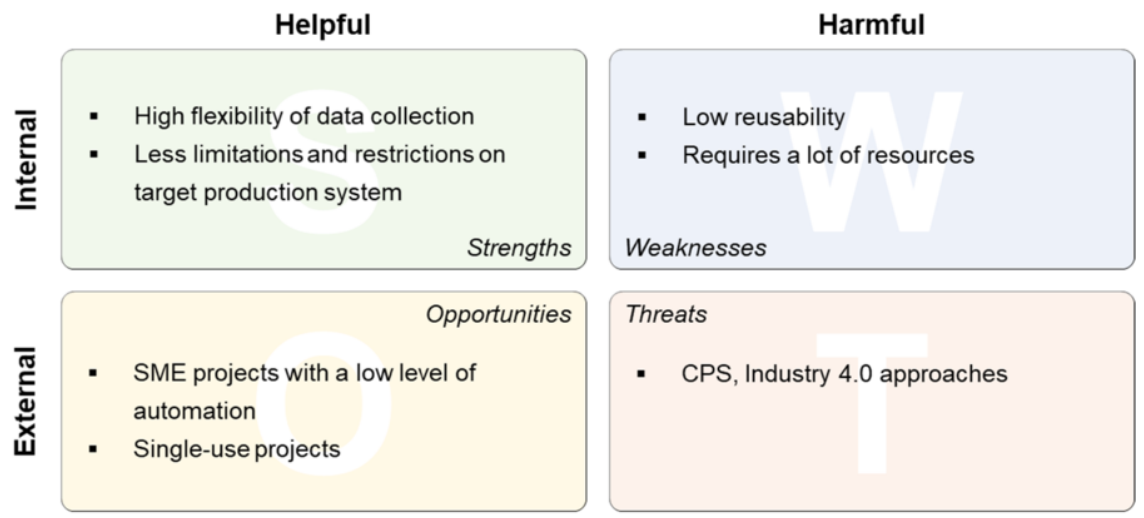

Figure 8 SWOT anaylsis of manufacturing simulation in a traditional approach

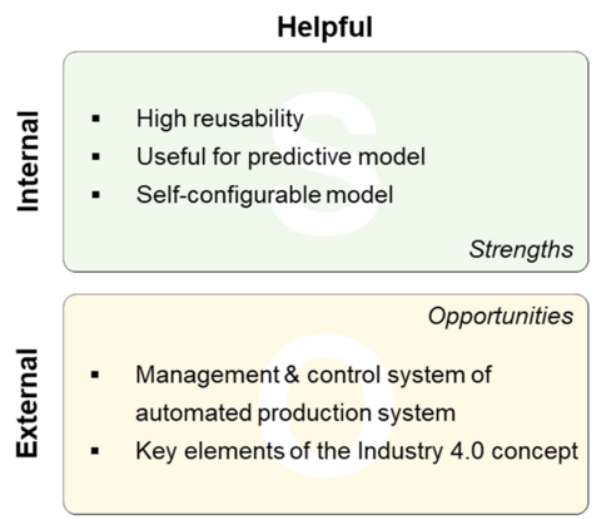

\begin{tabular}{|l|}
\multicolumn{1}{c}{ Harmful } \\
$\begin{array}{l}\text { - Requires infrastructure with data } \\
\text { collection, simulation modeling } \\
\text { and analysis }\end{array}$ \\
Weaknesses \\
\hline $\begin{array}{l}\text { Threats } \\
\text { - Stability and reliability of the system }\end{array}$ \\
\hline
\end{tabular}

Figure 9 SWOT anaylsis of manufacturing simulation in a CPS-based approach

\section{Conclusions}

In this research, a manufacturing simulation was analyzed and compared from both traditional approaches and CPS-based perspectives. The major components of datadriven manufacturing simulation were suggested by dividing into data gathering, modeling and simulation, and results analytics. The CPS-based approach to manufacturing simulation uses the real-time data collected from the production system to construct a simulation model and feedback the simulation results to the production system. Therefore, it is highly reusable and can be used in various fields such as monitoring, control, and prediction. However, this approach requires effective platforms for data collection, simulation modeling, and analysis. With the Industry 4.0 concept, manufacturing simulation will not not be a just single-use project to support decisionmaking, but rather a key contributor to overall production system operations. Nevertheless, traditional simulation approaches are flexible to apply. Therefore, it can be useful in production environments without infrastructure such as SMEs. 


\section{References}

[1] S. Wang, J. Wan, D. Li, and C. Zhang, Implementing Smart Factory of Industrie 4.0: An Outlook, International Journal of Distributed Sensor Networks, vol. 12, no. 1, 2016, pp. 1-10.

[2] R. Rosen, G. Von Wichert, G. Lo and K.D. Bettenhausen, About The Importance of Autonomy and Digital Twins for the Future of Manufacturing, IFAC-PapersOnLine, vol. 48, no. 3, 2015, pp. 567-572.

[3] Y. Liu and X. Xu, Industry 4.0 and Cloud Manufacturing: A Comparative Analysis, Journal of Manufacturing Science and Engineering, vol. 139, no. 3, 2017.

[4] F. Tao and M. Zhang, Digital Twin Shop-Floor: A New Shop-Floor Paradigm Towards Smart Manufacturing, IEEE Access, vol. 5, 2017, pp. 20418-20427.

[5] L. Barreto, A. Amaral and T. Pereira, Industry 4.0 implications in logistics: an overview, Procedia Manufacturing, vol. 13, pp. 1245-1252.

[6] V. Zeller, C. Hocken and V. Stich, acatech Industrie 4.0 Maturity Index - A Multidimensional Maturity Model, In: IFIP International Conference on Advances in Production Management Systems (APMS), Seoul, South Korea, 2018, pp. 105-113.

[7] F. Tao, Q. Qi, A. Liu and A. Kusiak, Data-driven smart manufacturing, Journal of Manufacturing Systems, vol. 48, 2018, pp. 157-169.

[8] C. Liu and P. Jiang, A Cyber-physical System Architecture in Shop Floor for Intelligent Manufacturing, Procedia CIRP, vol. 56, 2016, pp. 372-377.

[9] M. Schluse, M. Priggemeyer, L. Atorf and J. Rossmann, Experimentable Digital Twins - Streamlining Simulation-Based Systems Engineering for Industry 4.0, IEEE Transactions on Industrial Informatics, vol. 14, no. 4, 2018, pp. 1722-1731.

[10] Y.-T.T. Lee, F.H. Riddick and B.J.I. Johansson, Core Manufacturing Simulation Data - a manufacturing simulation integration standard: overview and case studies, International Journal of Computer Integrated Manufacturing, vol. 24, no. 8, 2011, pp. 689-709.

[11] S. Bergmann and S. Straßburger, On the Use of the Core Manufacturing Simulation Data (CMSD) Standard: Experiences and Recommendations, In: Fall Simulation Interoperability Workshop 2015, Orlando, Florida, USA, 2015.

[12] J. Lee, H.-A. Kao and S. Yang, Service Innovation and Smart Analytics for Industry 4.0 and Big Data Environment, Procedia CIRP, vol. 16, 2014, pp. 3-8.

[13] A. Radziwon, A. Bilberg, M. Bogers and E.S. Madsen, The Smart Factory: Exploring Adaptive and Flexible Manufacturing Solutions, Procedia Engineering, vol. 69, 2014, pp. 1184-1190.

[14] J. Xu, E. Huang, L. Hsieh, L.H. Lee, Q.-S. Jia and C.-H. Chen, Simulation optimization in the era of Industrial 4.0 and the Industrial Internet, Journal of Simulation, vol. 10, no. 4, 2016, pp. 310-320.

[15] A. Singh, F. Quint, P. Bertram and M. Ruskowski, A Framework for Semantic Description and Interoperatbility across Cyber-Physical Systems, International Journal on Advances in Intelligent Systems, vol. 12, no. 1, 2019, pp. 70-81. 